\title{
Properties of Zwitterionic Sulfobetaine Gels Containing Different Numbers of Methylene Units
}

\author{
Eva Oktavia Ningrum ${ }^{1, *}$, Shuji SAKOHARA ${ }^{2}$, Takehiko GOTOH ${ }^{3}$, Suprapto ${ }^{1}$, and Nurlaili Humaidah ${ }^{1}$ \\ ${ }^{1}$ Department of Industrial Chemical Engineering, Faculty of Vocational Studies, Institut Teknologi Sepuluh Nopember, Kampus ITS \\ Sukolilo, Surabaya, 60111, Indonesia \\ ${ }^{2}$ National Institute of Technology Niihama College, Yagumocho, 7-1, Niihama-shi, Ehime Prefecture 792-8580 \\ ${ }^{3}$ Department of Chemical Engineering, Graduate School of Engineering, Hiroshima University, Kagamiyama 1-4-1, Higashi- \\ Hiroshima, 739-8527, Japan
}

\begin{abstract}
The present research was performed aiming to develop gel with the characteristic of reversible thermosensitive in adsorbing heavy metal ions from its solution. There were three gels used in this study to adsorb heavy metal ion from salt solution provided $\mathrm{Zn}\left(\mathrm{NO}_{3}\right)_{2}$; copolymer gel consisting of zwitterionic betaine $N, N$-dimethyl(acrylamidopropyl)ammonium propane sulfonate (DMAAPS), N,N-dimethyl(acrylamidopropyl)ammonium butane sulfonate (DMAABS), and DMAAPS copolymerized with thermosensitive $\mathrm{N}$-isopropylacrylamide (NIPAM) gels. The aforementioned gels were employed to examine its ability in adsorbing and swelling after being synthesized through free radical polymerization before being affected by methylene spacer number, copolymerization, and temperature given. This research found that as the temperature increases, the ability of the gel in adsorbing the ions decreases. In the case of DMAAPS and DMAABS gel, the swelling degree value increases when the temperature also increases. Meanwhile, sulfobetaine which has larger spacer has higher adsorption ability but not with its swelling degree. In spite of having more spacer than DMAAPS, DMAABS found to have the smallest swelling degree value. In addition, NIPAM-co-DMAAPS was found to have both the highest adsorption ability and swelling degree value. Even though copolymer has less amount of sulfobetaine than DMAAPS and DMAABS gels', the copolymer gel was found to have higher ability of adsorption as many as ten times of other gels.
\end{abstract}

\section{Introduction}

The use of zwitterionic in polymer thermosensitive polymer copolymerization technique has become popular to be used as the alternative technique in several applications including biosensors, drug delivery system, catalysts, and separation media (Liu et al., 2010; Ningrum, 2019). Research has been previously conducted aiming to remove $\mathrm{Pb}^{2+}$ from mixed solutions of $\mathrm{Pb}^{2+} / \mathrm{Cu}^{2+}$ using zwiterionic hybrid polymer according to the polymerization reaction of ring-opening of pyromellitic dianhydride (PMDA) and sol-gel process by Liu et al. (Liu et al., 2005). There are three types of zwitterionic polymer, including sulfobetaine, phosphobetaine and carboxybetaine which consist of both anionic and cationic groups. The anionic group includes sulfonate, phosphate and carboxylate, while the cationic group includes quartenery ammonium (Kudaibergenov et al., 2006). The examples of zwitterionic sulfobetaine polymers are $N, N$ dimethyl(acrylamidopropyl)ammonium propane sulfonate (DMAAPS) and $N, N^{\prime}$-dimethyl(acrylamidepropyl)ammonium butane sulfonate (DMAABS).
A previous study was conducted by Ningrum et al (Ningrum et al., 2015) aiming to investigate the relationship between ion ability in adsorption and swelling properties on sulfobetaine gel. When the gel polymer concentration was higher than $180 \mathrm{~g} / \mathrm{l}$ and the swelling degree is low, no change was occurred at the amount of $\mathrm{Zn}^{2+}$ ions absorbed. Meanwhile, when the concentration was lower than $180 \mathrm{~g} / \mathrm{l}$ and the $\mathrm{s}$

welling degree was high, there is a decrease in the amount of $\mathrm{Zn}^{2+}$ ion absorbed. Meanwhile, the amount of $\mathrm{Zn}^{2+}$ ion adsorbed in the gel increased as $\mathrm{Zn}\left(\mathrm{NO}_{3}\right)_{2}$ concentration increases and the gels involved had the same swelling degree.

There are various previous researchers conducted aiming to investigate the copolymerization of sulfobetaine zwitterionic polymer and thermosensitive gel by several researchers. However, most of them only encouraged on the copolymer gel's synthesis and properties. The result of one of the previous research carried out by Ningrum et al. (Ningrum et al., 2014) obtained the relationship between the swelling degree properties and DMAAPS gel in adsorbing ions in various solutions as well as how the concentration of the cross-linker affects the adsorption

\footnotetext{
* Corresponding author: eva-oktavia@chem-eng.its.ac.id
} 
and the swelling of DMAAPS gel. The result obtained the relationship between the cross-linker concentration and DMAAPS gel adsorption ability in which the higher the cross-linker concentration, the better the DMAAPS gel in ion adsorption.

Thus, the purpose of this research was to investigate the spacer (methylene unit) amount within the sulfobetaine charged group in affecting the adsorption and swelling degree of the gel. Moreover, this research also aimed to know the comparison of the DMAAPS gel adsorption amount and copolymerization of DMAAPS with thermosensitive NIPAM at various cross-linker concentrations.

\section{Experimental Section}

This research used primary monomer in the form of $\mathrm{N}$ isopropylacrylamide (NIPAM) obtained from KJ Chemicals Co., Ltd., Japan that has been purified through recrystallization using $\mathrm{N}$-hexane. $\mathrm{N}, \mathrm{N}$-dimethyl(acrylamidopropyl)ammonium propane sulfonate (DMAAPS) was synthesized through ring-opening reaction (Ningrum et al., 2019) which was introduced by Lee and Tsai (Lee and Tsai, 1994). The reaction was carried out using $\mathrm{N}, \mathrm{N}$-dimethylaminopropylacrylamide (DMAPAA) obtained from KJ Chemicals Co., Ltd., Japan. and 1,3-propanesultone (PS) or 1,4-butanesultone (BS) obtained from Tokyo Chemical Industry Co., Ltd., Japan. Then, $100 \mathrm{~g}$ of DMAPAA and $200 \mathrm{~g}$ of acetonitrile was mixed and added by a dropwise of the mixture solution of PS or BS (75 g) and acetonitrile (75 g) for 90 minutes. The mixture of the solutions was then stirred at $30^{\circ} \mathrm{C}$ and then the stirring was continued for $16 \mathrm{~h}$. After the stirring, DMAAPS or DMAABS crystal was then washed using $500 \mathrm{~mL}$ of acetone. After washed, the stirring was continued for 2 days at room temperature. After the stirring, white crystals formed which were then filtered and washed using $500 \mathrm{~mL}$ of acetone. Drying of the product was then performed using vacuum oven at the temperature of $50^{\circ} \mathrm{C}$ for 24 hours.

NIPAM-co-DMAAPS synthesis was carried out through free radical polymerization reaction. For the reaction, N-isopropylacrylamide (NIPAM), DMAAPS, $N, N^{\prime}$-methylenebisacrylamide (MBAA) and $N, N, N^{\prime}, N^{\prime}-$ tetramethylethylenediamine (TEMED) were dissolved into distilled water until the volume of the total solution reaches $100 \mathrm{~mL}$. The dissolved oxygen was removed through purging process in the solution using nitrogen gas for 10 minutes. A solution of $20 \mathrm{ml}$ ammonium peroxodisulfate (APS) that has been purged previously was added to the monomer solution. The polymerization reaction occurred at $10^{\circ} \mathrm{C}$ for $6 \mathrm{~h}$ in flowing $\mathrm{N}_{2}$ gas. The gel synthesis is described in the table as follows:

Table 1. Gel's Synthesis Condition

\begin{tabular}{ccc}
\hline & Monomer & $\begin{array}{c}\text { Concentration } \\
(\mathbf{m m o l} / \mathbf{L})\end{array}$ \\
\hline NIPAM-co-DMAAPS & NIPAM & 800 \\
DMAAPS & DMAAPS & 200 \\
& DMAAPS & 500
\end{tabular}

\begin{tabular}{ccc} 
DMAABS & DMAABS & 500 \\
Linker & MBAA & 10,30 \\
Accelerator & TEMED & 10 \\
Initiator & APS & 2 \\
\hline
\end{tabular}

A separable four neck flask was used by placing a glass tube of $3 \mathrm{~mm}$ (ID) and $2 \mathrm{~cm}$ (L) in order to carry out the gel synthesis to obtain the cylinder gel. The polymerization reaction which results in the formation of the gel lasted for $6 \mathrm{~h}$. The gels formed was then cut until the length becomes $3 \mathrm{~mm}$. Distilled water was then used to wash the NIPAM-co-DMAAPS gel. After the washing, the gel was dried on Teflon paper spread on the petridish for several days. The petridish covered by plastic films with small holes aiming to reduce evaporation. The evaporation was reduced so that the drying process is slower and the gel does not crack. The other gels product was also made into small pieces, washed and dried using oven. After the drying, the gels were then mixed and the gels higher than 90 mesh size were chosen through sieving. There were two gels produced, cylinder gels used for the swelling test and the gels higher than 90 mesh size used for the gels adsorption tests. The concentration ions in the solution was obtained through Atomic Absorption Spectrophotometry (AAS) analysis.

\subsection{Swelling degree test (SD)}

The swelling degree (SD) test was conducted by letting the cylinder gels left for $12 \mathrm{~h}$ until it reached its equilibrium swelling. However, before and after reached the equilibrium swelling, its diameter was measured using milimeter block of $70^{\circ} \mathrm{C}$. The solution temperatures for this study were $10^{\circ} \mathrm{C}, 30^{\circ} \mathrm{C}, 50^{\circ} \mathrm{C}$, and $70^{\circ} \mathrm{C}$. The following equation was used to calculate the swelling degree

Swelling Degree $=\frac{d_{\text {swell }}{ }^{3}}{d_{d r y^{3}}}$

The $d_{\text {swell }}$ shows the gel diameter when it reached equilibrium swelling at a certain temperature (diameter of swollen gel) while $d_{\text {dry }}$ shows the dry gel diameter (dry gel).

\subsection{Adsorption test}

In the adsorption test, $\mathrm{ZnNO}_{3}$ solution was used. In this case, $20 \mathrm{ml}$ of $\mathrm{ZnNO}_{3}$ solution in the desired concentration was added by one gram of copolymer gel. The mixing was placed in a glass bottle put into a waterbath and stirred for $12 \mathrm{~h}$ at the desired temperature in order to achieve equilibrium adsorption conditions. Centrifuge was used to separate the gel from the solution in order to calculate the concentration of cations and anions in the solution after the adsorption process. Then the gel was filtered with a syringe filter.

The following equation was used to calculate the amount ions adsorbed into the gel calculated from the concentration of cations and anions before and after the adsorption process,

$Q=\frac{\left(C_{0}-C\right) V}{m}$ 2 
The Q shows the number of cations or anions adsorbed or decanted, $\mathrm{C}_{0}$ is the ions' concentration before the adsorption process in the solution, $\mathrm{C}$ is the ions' concentration after the adsorption process in the solution, while $\mathrm{V}$ is the volume of the solution and $\mathrm{m}$ is the dry gel weight $(\geq 90$ mesh). The ion concentration was determined using Atomic Absorption Spectophotometry (AAS) analysis.

\section{Result and Discussion}

\subsection{The Effect of Time on Gels' Adsorption Ability}

The effect of the gel adsorption's time length on the amount of $\mathrm{Zn}^{2+}$ ions adsorbed in $\mathrm{Zn}\left(\mathrm{NO}_{3}\right)_{2}$ solution in the time range of 0-24 $\mathrm{h}$ is shown in Figure 1 From this figure, the time of NIPAM-co-DMAAPS gel in achieving its equilibrium adsorption in $\mathrm{Zn}\left(\mathrm{NO}_{3}\right)_{2}$ solution can be determined. In the first $6 \mathrm{~h}$ the amount of $\mathrm{Zn}^{2+}$ ions adsorbed increased significantly. However, later it showed a slight decrease in the next 6-12 h continued with a constant. The phenomenon is because a large concentration gradient between the copolymer gel in the first $6 \mathrm{~h}$, so that the mass driving force before $6 \mathrm{~h}$ is greater than the driving force over $6 \mathrm{~h}$. After the first $6 \mathrm{~h}$, the ions adsorbed causing the concentration gradient decreases and the mass transfer rate becomes slower until finally reaches an equilibrium condition. The adsorption equilibrium achieved by the gel copolymers is above $12 \mathrm{~h}$ which will later be used in subsequent experiments (Ningrum et al., 2015).
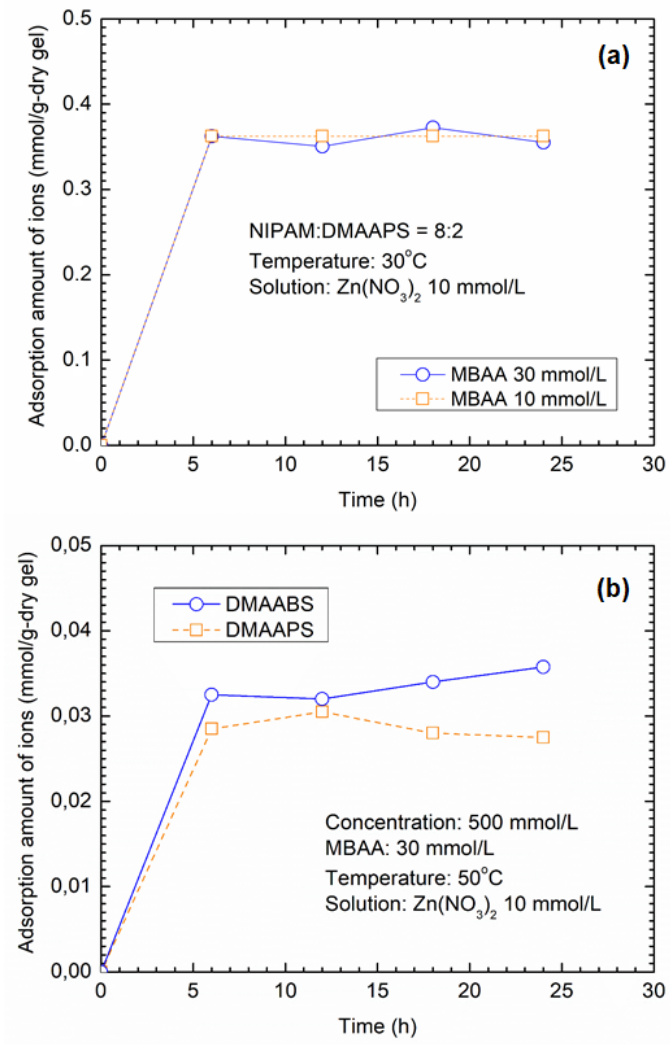

Figure 1. The effect of contact time on the adsorption ability of a) NIPAM-co-DMAAPS gels at $30^{\circ} \mathrm{C}$; b) DMAAPS and DMAABS gels at $50^{\circ} \mathrm{C}$

\subsection{The effect of temperature on gels adsorption ability}

Effect of temperature on the adsorption ability copolymers gel is shown in Figure 2. In all gels, as the temperature increased, the gels' adsorption ability of the ions decreased. The increase of the temperature was caused the bond between the ion and the charged group in the gel $\left(\mathrm{N}^{+}\right.$and $\left.\mathrm{SO}_{3}{ }^{-}\right)$weak so that the bond in the gel was dominated by intra-group, inter-chain, and intra-chain bonds. The number of ions adsorbed by DMAAPS gel (3 methylene spacer) increased with cross-linker concentration. The reason was due to in the gel having higher cross-linker concentration the bond formed was denser. This limits the gels' ability to expand and shrink. When the gel expands, the gel's swelling ability was limited by the bond formed from the polymer and crosslinker so that the distance between the charged groups was closer. Meanwhile, if the cross-linker concentration was low, the gel can expand better so that the distance between the charged groups was getting bigger. The distance between charged groups that are increasingly close together made it easier for intra-group bond (Ningrum et al., 2017a).

Ion adsorption on DMAABS gel (4 methylene spacer) is shown in Figure 2(b). In the figure, it can be seen that DMAABS gel adsorption ability was higher than the DMAAPS gel adsorption ability. This is in accordance with the initial hypothesis that further distance between the charged groups make the bonds between the charged groups weaker and cause the interaction within the gel between the solution ions and charged groups increased. There was no difference between the cross-linker gels with low and high concentration due to DMAABS properties which has hydrophobic properties (Ning et al., $2013 b$ ). Since the gel was more hydrophobic, the permeability of the gel was low so the solution was difficult to penetrate into the gel. The bonding between the charges in the gel with longer methylene spacers did not affect the flexibility of the charged group (Weers et al., 1991). Carboxybetaine molecule with methylene spacer molecules less than 3 between its charged groups have lower $\mathrm{pK}_{\mathrm{a}}$ compared to the molecule which has more than 3 methylence spacer. This indicates that carboxybetaine with methylene spacer $<3$ has a weak ability to interact with water compared to the carboxybetaine which has methylene spacer $>3$. In addition, between two charged groups in 1 molecule zwitterion separated by methylene spacer $>4$ did not affect each other with one another while in zwitterion, the charged groups are separated by methylene spacers $<3$, charged groups on the same molecule that are more interactive affect each other (Shao and Jiang, 2013). Looking at the similarities of sulfobetaine and carboxybetaine, it can be approximated that sulfobetaine with methylene spacer $>3$ (in this case DMAABS) is easier to bind to water so that it inhibited $\mathrm{Zn}\left(\mathrm{NO}_{3}\right)_{2}$ ions binding to charged groups $\left(\mathrm{N}^{+}\right.$and $\left.\mathrm{SO}_{3}^{-}\right)$. Poly (DMAABS) itself had an effective inter-chain pair 
formation in charged groups inside sulfobetaine. Because the inter-chain bonds occurred were very strong, the difference in cross-linker did not have any significant effect on gels' ion adsorption (Ning et al., 2013a).
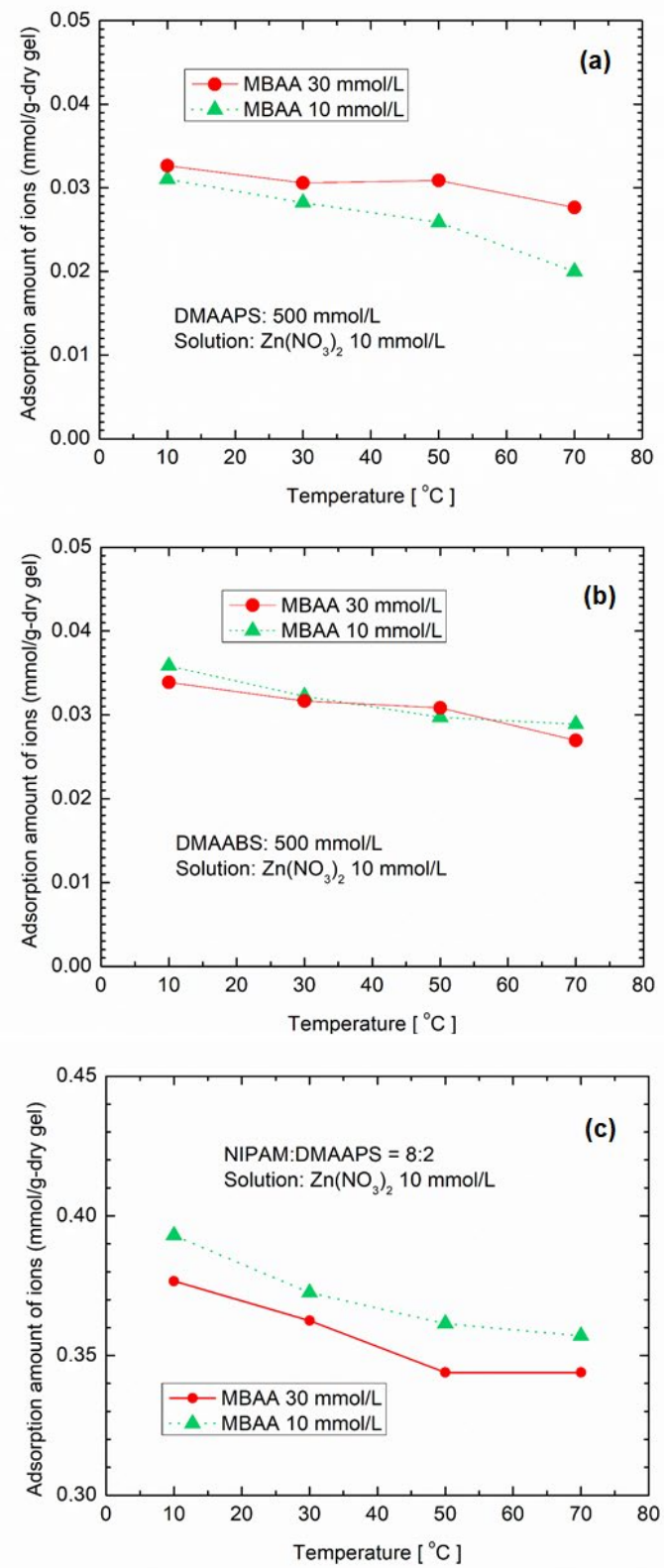

Figure 2. Effect of temperature on the adsorption ability of a) DMAAPS, b) DMAABS, and c) NIPAMco-DMAAPS gels

Temperature also affected the ions' adsorption ability, in which the more increase the temperature, the weaker the interaction of ions in the solution and the group of charged groups. In the case NIPAM-coDMAAPS, the copolymerization DMAAPS with thermosensitive NIPAM increased the gels' adsorption ability ten times as seen in Figure 2(c). The increase in adsorption ability was affected by the presence of NIPAM which acts as the separator between DMAAPS molecules. NIPAM is a thermosensitive polymer well at low temperatures (Ningrum et al., 2017b). When NIPAM expanded, the distance between DMAAPS molecules increased and the intra-chain, inter-chain and intra-group bonds decreased. With the decrease of the bond, the charged groups can bind ions in the solution. According to the figure above, it can be obtained that when there was an increase in the temperature, the gel adsorption ability decreases. At high temperatures, the adsorption ability of the gel was low, this was due to an increase in temperature which causes the interaction between solution ions and charged groups in the gel weaker. In addition, the reduced ability of the gel to adsorb ions was caused by gels that shrink at high temperatures so that the gel became denser. When shrink, the ions were pushed out from the gel because of the inter-chain, intra-chain, and intra-group bonds. In addition, thermal motion can also weaken the strength of inter ion bonds (Ningrum et al., 2014).

\subsection{The Effect of Temperature on Swelling Degree}

Figure 3 shows the effect of temperature on the gel's swelling degree. For DMAAPS and DMAABS, the gels' swelling degree increased as the temperature increased. However, when the temperature increased, the NIPAMco-DMAAPS gel's swelling degree decreased. This is because NIPAM concentration was higher than DMAAPS so that the nature of the copolymer gel was dominated by NIPAM property. The higher the concentration of the cross-linker, the gel swelling degree decreased, as seen in the DMAAPS gel and NIPAM-co-DMAAPS gel. The high cross-linker concentration caused the gel stiff. Because the polymer is in the form of a gel, when the chain expanded, the entire bond swells. However, because of the higher cross-linker, there is a limitation on the gel's swelling ability. The cross-linker concentration has no significant effect in DMAABS gel. This is because the bonds between molecules are strong and the number of inter-chain bonds present in DMAAPS is high so that the polymer chain entangle (Ning et al., 2013a). 

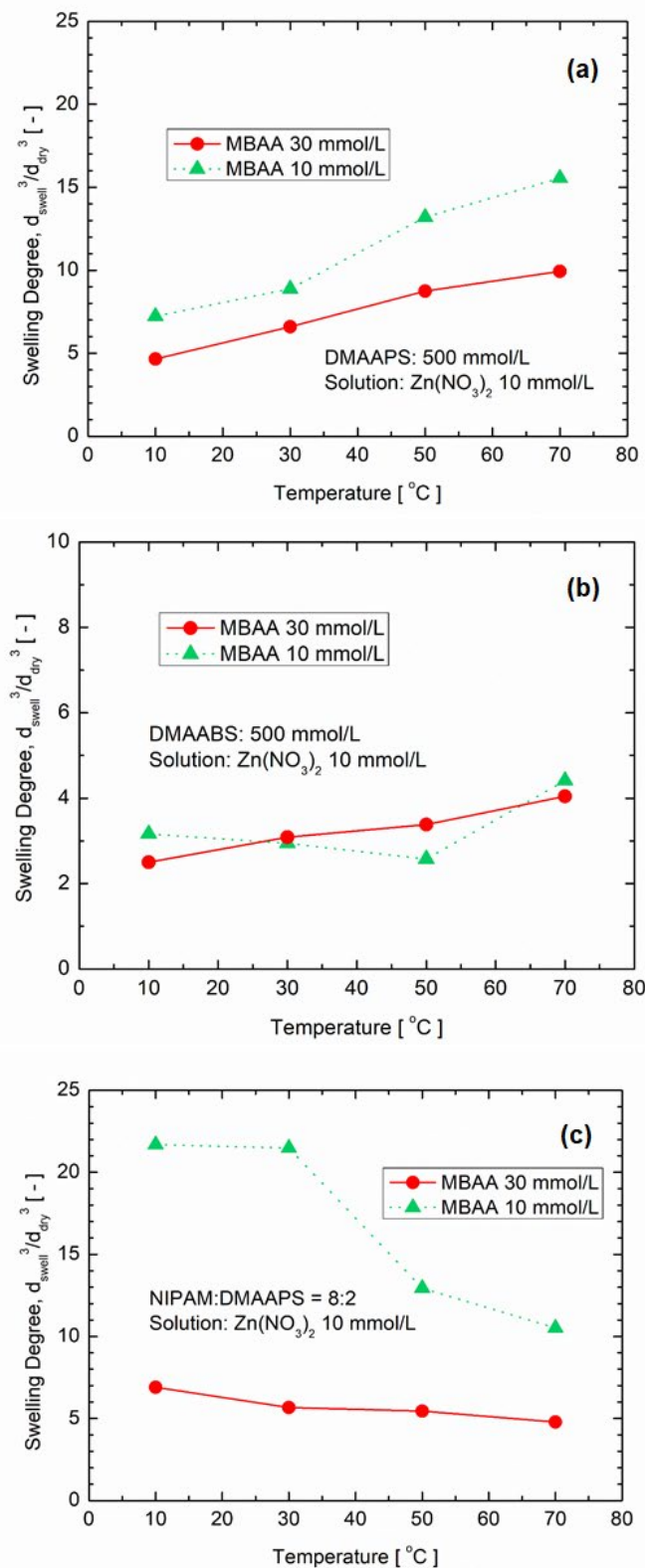

Figure 3. The Effect of temperature on the swelling degree of a) DMAABS, b) DMAAPS, and c) NIPAM-co-DMAAPS gels

\section{Conclusion}

The equilibrium adsorption of the sulfobetaine gel and its copolymerization with NIPAM was achieved above $6 \mathrm{~h}$. The cross-linker has a significant effect on swelling degree of the DMAAPS and NIPAM-co-DMAAPS gels where the addition of the cross-linker concentration decreases the swelling degree value. However, in DMAABS gel, the addition of cross-linker did not have any significant effect on the swelling degree value. The ability of NIPAM-co-DMAAPS adsorption increased by the decrease of the cross-linker's concentration, while in DMAAPS and DMAABS the change in cross-linker concentration did not have any significant effect on the gel adsorption ability. NIPAM-co-DMAAPS gel had the highest adsorption value which was then followed by DMAABS gel and the lowest was DMAAPS gel. The adsorption ability of the gel both NIPAM-co-DMAAPS, DMAAPS, and DMAABS decreased as the temperature increases as well. In the case of swelling degree, NIPAMco-DMAAPS has the highest swelling degree followed by DMAAPS and the lowest was DMAABS. As the temperature increased, the NIPAM-co-DMAAPS gel's swelling degree also decreased. Meanwhile, as for the DMAABS and DMAAPS gel, their swelling degree increased as the temperature increased.

\section{Acknowledgements}

This research was financially supported by research grant of Penelitian Kerjasama Luar Negeri 2019 (853/PKS/ITS/2019) from Direktorat Jendral Penguatan Riset dan Pengembangan Kemenristekdikti.

\section{References}

Kudaibergenov, S. E., W. Jaeger, and A. Laschewsky; "Polymeric Betaines: Synthesis, Characterization, and Application," Adv. Polym. Sci., 201, 157-224 (2006)

Lee, W.-F. and C.-C. Tsai; "Synthesis and Solubility of the Poly(sulfobetaine)s and the Corresponding Cationic Polymers: 1. Synthesis and Characterization of Sulfobetaines and the Corresponding Cationic Monomers by Nuclear Magnetic Resonance Spectra," Polymer, 35, 2210-2217 (1994)

Liu, J., T. Xu, and Y. Fu; "Fundamental Studies of Novel Inorganic-Organic Zwitterionic Hybrids. 1. Preparation and Characterizations of Hybrid Zwitterionic Polymers," J. Non-Cryst.Solids, 351, 3050-3059 (2005)

Liu, J., Y. Ma, T. Xu, and G. Shao; "Preparation of Zwitterionic Hybrid Polymer and Its Application for the Removal of Heavy Metal Ions from Water,“ J. Hazard. Mater, 178, 1021-1029 (2010)

Ning, J., K. Kubota, G. Li, and K. Haraguchi; "Characteristics of Zwitterionic Sulfobetaine Acrylamide Polymer and the Hydrogels Prepared by Free-Radical Polymerization and Effects of Physical and Chemical Crosslinks on the UCST," React. Funct. Polym., 73, 969$978(2013 a)$

Ning, J., G. Li, and K. Haraguchi; "Synthesis of Highly Stretchable, Mechanically Tough, Zwitterionic Sulfobetaine Nanocomposite Gels with Controlled Thermosensitivities," Macromolecules, 46, 5317-5328 (2013b)

Ningrum, E. O.; "The Adsorption Behaviors of Thermosensitive poly (DMAAPS) Grafted onto EVA Porous Support," IOP Conference Series: Materials Science and Engineering, pp. 012037, IOP Publishing (2019)

Ningrum, E. O., Y. Murakami, Y. Ohfuka, T. Gotoh, and S. Sakohara; " Investigation of Ion Adsorption Properties 
of Sulfobetaine Gel and Relationship with Its Swelling Behavior," Polymer, 55, 5189-5197 (2014)

Ningrum, E. O., Y. Ohfuka, T. Gotoh, and S. Sakohara; "Effects of Specific Anions on the Relationship between the Ion-Adsorption Properties of Sulfobetaine Gel and Its Swelling Behavior," Polymer, 59, 144-154 (2015)

Ningrum, E. O., A. Purwanto, E. O. Mulyadi, D. I. Dewitasari, and S. Sumarno; "Adsorption and Desorption of $\mathrm{Na}^{+}$and $\mathrm{NO}_{3}{ }^{-}$Ions on Thermosensitive NIPAM-coDMAAPS Gel in Aqueous Solution," Indones. J. Chem., 17, 446-452 (2017a)

Ningrum, E. O., A. Purwanto, H. Ni'mah, S. Sumarno, D. I. Dewitasari, and E. O. Mulyadi; "Ion Adsorption and Desorption Behaviors of Thermosensitive NIPAM-coDMAAPS Gel by Temperature Swing," AIP Conf. Proc., 1840(1), 090007 (2017b)

Ningrum, E. O., S. Sakohara, T. Gotoh, Suprapto, and N. Humaidah; "The Effect of Cation and Anion Species on The Transition and Adsorption Behaviors of Thermosensitive Sulfobetaine Gel Based Adsorbent," Int. J. Technol., 10, 443-452 (2019)

Shao, Q. and S. Jiang; "Effect of Carbon Spacer Length on Zwitterionic Carboxybetaines," J. Phys. Chem. B, 117, 1357-1366 (2013)

Weers, J. G., J. F. Rathman, F. U. Axe, C. A. Crichlow, L. D. Foland, D. R. Scheuing, R. J. Wiersema, and A. G. Zielske; "Effect of the Intramolecular Charge Separation Distance on the Solution Properties of Betaines and Sulfobetaines," Langmuir, 7, 854-867 (1991) 